\title{
Magnitude and Factors Affecting Virological Treatment Failure among HIV Reactive Adults from Selected Hospitals of North Shoa Zone, Amhara Region, Ethiopia
}

Behailu Tariku Derseh ( $\nabla$ minastariku@gmail.com )

Debre Berhan University

Belay Shewayerga

Debre Berhan University

Abinet Dagnaw

Debre Berhan University

Elyas Admasu Basha

Debre Berhan University

Research

Keywords: Adherence, First-line ART, virologic treatment failure, North Shoa, Ethiopia

Posted Date: August 27th, 2020

DOI: https://doi.org/10.21203/rs.3.rs-64123/v1

License: (c) (i) This work is licensed under a Creative Commons Attribution 4.0 International License.

Read Full License 
3 Behailu Tariku Derseh ${ }^{1 *}$, Belay Shewayerga $^{1}$, Abinet Dagnaw $^{1}$, Elyas Admasu Basha ${ }^{2}$

$4{ }^{1}$ Department of Public Health, College of Health Sciences, Debre Berhan University, Ethiopia.

$5{ }^{2}$ Department of Nursing, College of Health Sciences, Debre Berhan University, Ethiopia.

6 Corresponding author: Behailu Tariku Derseh (minastariku@gmail.com)

$7 \quad$ Email address

8 Belay Shewayerga (belayshewayrga@gmail.com)

9 Abinet Dagnaw (abinetdagn@gmail.com)

10 Elyas Admasu Basha (admasu.elias5@gmail.com)

\section{Abstract}

Background: Nowadays Human Immuno-deficiency Virus (HIV) is one of the devastating and prevalent viruses affecting the globe without a cure. Highly Active Antiretroviral Therapy (HAART) significantly reduced the morbidity and mortality of patients with HIV infection. Although there is increasing global use of antiretroviral therapy (ART) for the treatment of HIV/AIDs, the global trends in ART failure are growing. In developing countries including Ethiopia, particularly in our study settings, information related to the magnitude and correlates of virological treatment failure is scarce. Thus, this study aimed at assessing the magnitude of virological treatment failure and associated factors among HIV reactive adults at selected hospitals of North Shoa, Amhara Region, Ethiopia.

Methods: a facility-based cross-sectional study was conducted among 498 study participants who started their first-line HAART from August 2005 to December 2018. Data were collected from patients' charts and face-to-face interviews using a structured questionnaire. The bivariable analysis was executed to select candidate predictor variables at a p-value of less than 0.2. 
Multivariable logistic regression (forward stepwise, conditional) analysis was used to identify factors associated with virologic failure at a significant level of 5\%. Model adequacy check was done by Hosmer and Lemeshow $(\mathrm{p}=0.57)$ and Naglkerke R Square $(0.46)$ was calculated to express the variability of virological failure by predictors.

Result: More than half $290(58.2 \%)$ of the study participants were females. The median age at ART initiation was 40 years with an interquartile range (IQR) of 15 years. The median duration of virologic failure since initiation of treatment was 96 months (IQR) of 72. The magnitude of virological treatment failure was $10.24 \%$ (95\% CI: 7.57\% - 12.91\%). Poor ART drug adherence $(\mathrm{AOR}=4.54 ; 95 \% \mathrm{CI}: 2.09,9.87), \mathrm{CD} 4$ count less than 250 cell $/ \mu \mathrm{l}(\mathrm{AOR}=24.88 ; 95 \% \mathrm{CI}$ : 11.73, 52.81) and Poor Quality of Life (QoL) $(\mathrm{AOR}=2.65 ; 95 \%$ CI: 1.12, 6.25) were independent predictors of virologic failure.

Conclusion: The magnitude of Virologic ART treatment failure in this study was relatively high. Poor ART drug adherence, patients' having lower CD4 count and poorer quality of life were predictors of treatment failure. Thus, an intervention programs that enriches patients' health-related quality of life should be implemented. Moreover, counseling that supplements the importance of drug adherence and reduction of risks that lower CD4 counts should be given emphasis which in turn helps to prevent first-line ART treatment failure.

Keywords: Adherence, First-line ART, virologic treatment failure, North Shoa, Ethiopia

\section{Background}

HIV continues to be a major global public health problem. In 2016, an estimated 36.7 million people living with HIV, among this 34.5 million were adults and 2.1 million were children, with a global HIV prevalence of $0.8 \%$ among adults. There were about 1.8 million new HIV infections - a decline from 2.1 million new infections in 2015. The majority of people living with HIV located in low and middle-income countries, with an estimated 25.5 million living in sub- 
Saharan Africa. Among this group, 19.4 million are living in East and Southern Africa this year

50 (1, 2). In 2016 around 1 million people died with AIDS-related illnesses compared to 1.9 million in 2005 and 1.5 million in $2010(1,3)$.

52 Globally, 20.9 million [18.4 million-21.7 million] people living with HIV were accessing antiretroviral therapy in June 2017. In 2016, around 53\% [39-65\%] of all people living with HIV had access to treatment. Some 54\% [40-65\%] of adults aged 15 years and older living with HIV had access to treatment. In eastern and southern Africa, 11.7 million people were accessing antiretroviral therapy, 60\% [48-68\%] of all people living with HIV in the region in 2016. In Ethiopia, 420 000, 59\% [47\%-73\%] people were accessing antiretroviral therapy in 2016, among this 399000, 61\% [49\%-75\%] adults aged 15 years and older living with HIV had access to treatment $(1,4)$.

WHO recommendation to "treat all" principles to reach a total of 36.7 million people who must be successfully maintained on treatment for life. The global expansion of antiretroviral therapy has been the primary contributor to a $48 \%$ decline in deaths from AIDS-related causes, from a peak of 1.9 million [1.7 million-2.2 million] in 2005 to 1.0 million [830 $000-12$ million] in $2016(5,6)$. With this scale-up of ART coverage, an increasing proportion of people initiating ART are likely to be infected with a virus that is resistant to one or more WHO-recommended first-line ARV drugs (7).

67 According to the WHO definition, ART failure is defined as clinically (New or recurrent clinical event), immunologically (CD4 count falls to the baseline) and virological failure (viral load above 1000 copies/ml based on two consecutive viral load measurements after 3 months with adherence support), after 6 months of Effective treatment (8). WHO's Report on HIV drug resistance (HIVDR) in 2017 demonstrates a steady increase in the prevalence of HIVDR in

72 people initiating first-line ART since 2001, most notably in Southern and Eastern Africa. The

73 prevalence of HIVDR in people initiating first-line ART was 6.8\% in 2010, and estimates from 74 recent nationally representative surveys show levels of HIVDR above 10\% (5). Currently, WHO 75 recommended first-line ART includes Tenofovir (TDF), Efavirenz (EFV) combined 76 with Lamivudine(3TC), and Zidovudine (AZT) alternatives for TDF and Nevirapine (NVP), 77 Dolutegravir ( DTG )as alternatives for EFV (8). 
The treatment of people with this first-line antiretroviral (ARV) drugs will inevitably be accompanied by the emergence and transmission of drug-resistant virus. HIVDR limits treatment options and may necessitate a switch to more expensive regimens ( $2^{\text {nd }}$ line ART) which associated with greater long-term toxicity. Moreover, significant population-levels of treatment failure may lead to an increase in HIV/AIDS-related morbidity and mortality $(8,9)$.

In Ethiopia, 718,500 people were living with HIV/AIDS in 2016, of these, 653,412 were adults and 65,088 were children under 15 years of age, adult HIV prevalence was 1.18 this year (3). Antiretroviral Therapy (ART) service began in August 2003 with payment and free ART launched in January 2005. Ethiopia has already adopted major strategies of WHO guidance to meet the third 90 targets (90\% of people on treatment are virally suppressed). In 2016 a total of 420,000 people living with HIV were put on anti-retroviral treatment and virological suppression rates were $51 \%$ at the national level $(4,10)$. Currently, patients on HAART are monitored with viral load, immunological and clinical assessment among this viral load monitoring is the gold standard method to diagnose ART failure. Though WHO's recommendation to "treat all" living with HIV immediately after confirming HIV diagnosis that helps to reduce morbidity levels and premature death and the continued expansion of ART coverage, there were an increasing proportion of people on ART had HIV drug resistance (HIVDR); like Pretreatment HIV drug resistance (PDR) and acquired HIV drug resistance (ADR) to one or more of first-line ARV drugs $(10,11)$.

The human cost of HIVDR cannot be underestimated. HIV drug resistance associated with increased mortality and reduced effectiveness of treatment regimens. So preventing, monitoring and responding to HIVDR is therefore critical to maintaining current achievements, improving patient outcomes, and guarantee the long term sustainability of care and treatment programs (7). Studies have been conducted from different countries to identify the magnitude and factors associated with first-line ART a failure like in low-income and middle-income countries, a systematic review and meta-regression analysis, which indicate that the prevalence estimate of first-line ART resistance in 2016 was $11 \%$ in southern Africa, 10.1\% in eastern Africa, $7.2 \%$ in western and central Africa, and 9.4\% in Latin America and the Caribbean (12). 
According to a study conducted in Tanzania, more than $50 \%$ of the patients on first-line ART were experiencing immunological failure. Another study conducted in the Tigray region in 2017 virological and immunological failure in the study area were $11.5 \%$ and $6.5 \%$, respectively(13, 14), $29 \%$ in Colombia (15), $74 \%$ in Sweden (Hunduran) (16), $28 \%$ in China (17). However, in our study settings, studies which assess the association between virologic failure and healthrelated quality of life among ARV clients were scarce. Therefore, this study was aimed to assess the magnitude and predictors of virologic treatment failure among adult people with first-line ART.

\section{Methods}

\section{Study Area and Setting}

Currently, there are eight public hospitals, one private hospital, and more than ten Health center that providing ART services in North Shoa zone. Among these eight public hospitals, four district hospitals started ART services recently. The study was conducted in high caseload health institutions; Debre Berhan referral hospital and Mehal Meda District hospital, both in north Shoa zone, Amhara region, Ethiopia. According to the monthly report of Debre Berhan hospital, as of December 2018, in Debre Berhan referral hospital ever enrolled HIV positive clients to chronic HIV care starting from September 2005-December 2018 is 5951, patients ever started on Highly Active Antiretroviral Therapy (HAART) is 3834 and total current on ART is 1712 among this who's age $\geq 18$ years is $1651(\mathrm{~F}=1067, \mathrm{M}=584)(18)$. In Mehal Meda Hospital the number of patients ever enrolled in HIV chronic care starting from September 2005December 2018, is 1005, patients ever started on Highly Active Antiretroviral Therapy (HAART) is 767 and total current on ART is 448 among this who's age $\geq 18$ years is 411 $(\mathrm{F}=246, \mathrm{M}=165)(19)$.

\section{Study Design and Period}

A facility based cross-sectional study design was conducted among HIV-positive patients on HAART for $\geq 6$ months to test the size of first-line ART failure and its associated factors from January 28/2019 - March 22/2019. 
133 Source and study population

134 The source population was all human immunodeficiency virus (HIV) infected clients with age $135 \geq 18$ yrs who were on first-line Antiretroviral Therapy (ART) regimen in Debre Berhan referral 136 Hospital and Mehal-Meda district Hospital. The study population were all HIV/AIDS clients 137 who enrolled in the HAART program, who meet the inclusion criteria and available during the 138 study period to get service from the ART unit.

\section{Sample size determination and sampling procedure}

140 The sample size is determined based on a single population proportion formula. The proportion 141 of first-line Anti-retroviral Treatment Failure is taken from a study done in Ethiopia (14). Where, $142 \mathrm{p}=0.115$, confidence interval of $95 \%\left(\mathrm{Z} \frac{\boldsymbol{a}}{2}\right.$, critical value 1.96$)$, with a marginal error of $4 \%$ and

$14310 \%$ non-response rate. The sample size (n) is calculated as; $n=\left(z \frac{\boldsymbol{a}}{2}\right)^{2} * \frac{\boldsymbol{p}(\mathbf{1}-\boldsymbol{p})}{\boldsymbol{d}^{2}}=(\mathbf{1 . 9 6})^{\mathbf{2}}$ $144 * \frac{\mathbf{0 . 1 1 5}(\mathbf{1}-\mathbf{0 . 1 1 5})}{(\mathbf{0 . 0 4} \times \mathbf{0 . 0 4})^{2}}=244$ by considering $10 \%$ non-response rate, and multiplying it by a design 145 effect of 2, the total sample size estimated was 538.

146 A cluster sampling technique was used to select hospitals. There are eight public hospitals in 147 North Shoa Zone. Out of eight ART-site public hospitals, four hospitals were selected by the 148 number of cases they have and late initiation of ART. Then, Debre Berhan referral hospital and 149 Mehal-Meda district hospital were selected by a simple random sampling technique from four 150 hospitals.

151 Finally, consecutive sampling technique was used to select 538 study participants from two HIV 152 care center clinics, by allocating total sample size proportionally based on the number of clients/ 153 patients they have. 


\section{Operational definition}

155 Adherence: the extent to which a person's activities, taking medications, following corresponds

156 with accepting instructions from a health care provider (20).

157 Good adherence: if a client used greater than or equal to $95 \%$ adherence, that is, missing only 1 158 out of 30 doses or missing 2 from the 60 doses $(8)$.

159 Fair adherence: if a client used 85-94\% adherence, meaning, missing 2-4 doses out of 30 doses 160 or 4 to 9 doses from $60 \operatorname{doses}(8)$.

161 Poor adherence: if a client used less than $85 \%$ adherence, that is, missing $\geq 5$ doses out of 30 162 doses or more than 10 doses from 60 doses (8).

163 Virological failure: is considered in this study, if a patient has virological failure. That is viral 164 load above 1000 copies/mL based on two consecutive viral load measurements in 3 months, with 165 adherence support following the first viral load test (8).

166 Immunological failure: CD4 count at or below 250 cells/mm3 following clinical failure or 167 Persistent CD4 levels below 100 cells/mm3(8).

168 Clinical failure: New or recurrent clinical event indicating severe immunodeficiency after 6 169 months of effective treatment(8).

170 Data collection tool and procedure

171 Data collection was conducted by using a structured questionnaire and structured checklist to 172 collect data from patient follow-up form, ART register, and the electronic database for ART 173 program. Relevant clinical data such as CD4 count, clinical stage, HAART regimen, and drug 174 adherence status were extracted from participants' medical charts. Data on supply disruptions of 175 HIV-commodities like ART regimens were interviewed by the clients and from existing 176 registers. In addition, all selected sampled clients who come to ART follow-up clinics were 177 interviewed to collect socio-demographic information and drug adherence level. The 
178 questionnaire was prepared in the English language and translated into Amharic and back to 179 English to confirm the consistency questionnaire and checklist. Data were collected by two health information technology (HIT) personnel's that have diploma and experience in managing ART data. Two data collectors and two supervisors (BSC nurses) were trained for half a day about the aims of the study, the contents of tool, and how to collect the data before the data collection. Health-related Quality of Life (HRQoL) of the clients was measured to quantify multidimensional components of health perceived by clients in the past two weeks prior to data collection and includes; physical, mental, emotional, and social domains (21). The tool is known as WHOQOL-HIV BREF and developed by the World Health Organization; Mental Health Department. The questions are measured in 5-scales and we tested the reliability in our context (Cronbach $\alpha=0.727)$.

Data Processing and Analysis.

The data were checked for completeness and consistency during data collection. The data also cleaned during data entry into EPI info version 3.5.1 and has been transported into SPSS version 20. Data exploration technique was used to check the inconsistency and detect outliers in the dataset. Normality test was performed by using Shapiro-Wilk (P-Value > 0.05), KolmogorovSmirnova (P-Value > 0.05), and visual inspection of Q-Q Plot, P-P Plot, and Histogram. Descriptive statistics were used to express variables in terms of tables, percentages, and frequencies. The bivariable binary logistic regression was used to select predictor variables at a cut off points $(<0.2)$. Logistic regression assumptions (sample size and multicollinearity test) were used to check whether variables have satisfied the rules in regression or not. Variance Inflation Factor (VIF) > 10 was used to declare the presence and absence of multicollinearity. Forward stepwise conditional logistic regression analysis was used to determine the independent predictors of virologic failure. To calculate the measures of association, Adjusted Odds Ratio (AOR) with its 95\% Confidence Interval (CI) at 5\% level of significance was used. The model fitness test was performed by Hosmer and Lemeshow test $(\mathrm{p}=0.573)$ and model summary was done by Naglkerke $\mathrm{R}$ square (0.461) which express the variability of virologic treatment failure in terms of CD4 counts, Quality of Life and ART treatment drug adherence. 


\section{Result}

\section{Socio-demographic characteristics of patients}

209 A total of 498 HIV infected individuals on first-line ART regimens were voluntarily participated 210 in this study with a response rate of $92.5 \%$. The rest clients were refused to participate in the 211 study due to unwillingness. More than half 290 (58.2\%) of the study participants were female.

212 The median age of the participants was 40 with an interquartile range of (IQR $=15)$. Regarding

213 the educational level, $80(16.1 \%)$ of the study participants had no formal education, $209(42 \%)$ of 214 them completed primary school, $86(17.3 \%)$ completed college or university. Seventy-six $215(15.3 \%)$ were government employees and $230(46.2 \%)$ of them were self-employed. Two216 hundred sixty-one (52.4\%) of the study participants had a monthly income above 1000 per 217 month (Table 1).

218 Table 1. Socio-demographic characteristics of the respondents in Debre-Berhan referral hospital 219 and Mehal-Meda hospital, North Showa Zone, April/2019. (n=498)

\begin{tabular}{|l|l|l|}
\hline Variable & Frequency & Percent \\
\hline Sex & & \\
\hline Female & 290 & 58.2 \\
\hline Male & 208 & 41.8 \\
\hline Age(year) & & \\
\hline $18-30$ & 68 & 13.7 \\
\hline $31-45$ & 264 & 53.0 \\
\hline $46-60$ & 142 & 28.5 \\
\hline$>60$ & 24 & 4.8 \\
\hline Religion & & \\
\hline Orthodox & 465 & 93.4 \\
\hline Muslim & 19 & 3.8 \\
\hline Protestant & 14 & 2.8 \\
\hline Employment & & \\
\hline Employed full time & 76 & 15.3 \\
\hline Unemployed & 103 & 20.7 \\
\hline
\end{tabular}




\begin{tabular}{|l|l|lr|}
\hline Self-employed & 230 & 46.2 & 220 \\
\hline Farmer & 89 & 17.9 & 221 \\
\hline Level of education & & & \\
\hline Not educated & 80 & 16.1 & \\
\hline Elementary & 209 & 42.0 & \\
\hline Secondary & 123 & 24.7 & 223 \\
\hline College and above & 86 & 17.3 & \\
\hline Residence & & & 224 \\
\hline Urban & 418 & 83.9 & \\
\hline Rural & 80 & 16.1 & \\
\hline Monthly income & & & 226 \\
\hline$<=500$ & 82 & 16.5 & 31.1 \\
\hline $501-100$ & 155 & 52.4 &
\end{tabular}

\section{Baseline clinical characteristics of patients}

231 The majority, $434(87.1 \%)$ of study participants were working by their functional status and 482

$232(96.8 \%)$ of participants had hemoglobin measurement of greater or equal to $10 \mathrm{mg} / \mathrm{dl}$ at the start

233 of HAART. The median CD4 count was 184 cell/ $\mu \mathrm{l}(\mathrm{IQR}=182)$ and TB infection was confirmed

234 in $138(27.7 \%)$ since the start of HAART. Moreover, $190(38.2 \%)$ and $91(18.3 \%)$ of study

235 participants had started treatment with Tonofovir- Lamivudine-Efavirenz, and Zidovudine-

236 Lamivudine-Efavirenz regimen, respectively. In addition, one hundred sixteen (23.3\%) of them

237 have a history of malnutrition and 125 (25.1\%) of the participants were non-adhered to their

238 medication (Table 2). 
Table 2. Baseline and follow up characteristics of HIV/AIDS clients on antiretroviral therapy in

\begin{tabular}{|c|c|c|}
\hline Variable & Category & Frequency $(\%)$ \\
\hline \multirow[t]{6}{*}{ Baseline first-line HAART regimen } & $\mathrm{D} 4 \mathrm{~T}+3 \mathrm{TC}+\mathrm{NVP}$ & $67(13.5)$ \\
\hline & $\mathrm{D} 4 \mathrm{~T}+3 \mathrm{TC}+\mathrm{EFV}$ & $32(6.4)$ \\
\hline & $\mathrm{AZT}+3 \mathrm{TC}+\mathrm{NVP}$ & $82(16.5)$ \\
\hline & $\mathrm{AZT}+3 \mathrm{TC}+\mathrm{EFV}$ & $91(18.3)$ \\
\hline & $\mathrm{TDF}+3 \mathrm{TC}+\mathrm{EFV}$ & $190(38.2)$ \\
\hline & $\mathrm{TDF}+3 \mathrm{TPC}+\mathrm{NV}$ & $36(7.2)$ \\
\hline \multirow[t]{3}{*}{ Baseline WHO stages } & I & $398(79.9)$ \\
\hline & II & $70(14.1)$ \\
\hline & III & $30(6.0)$ \\
\hline \multirow{2}{*}{ Baseline hemoglobin level } & $<10$ & $16(3.2)$ \\
\hline & $\geq 10$ & $482(96.8)$ \\
\hline \multirow[t]{2}{*}{ History of TB } & Yes & $138(27.7)$ \\
\hline & No & $360(72.3)$ \\
\hline \multirow[t]{3}{*}{ Functional status } & Working & $434(87.1)$ \\
\hline & Ambulatory & $60(12.0)$ \\
\hline & Bed-ridden & $4(0.8)$ \\
\hline \multirow[t]{4}{*}{ Baseline CD4 results } & $\leq 100$ & $107(21.5)$ \\
\hline & $101-350$ & $306(61.4)$ \\
\hline & $351-500$ & $45(9)$ \\
\hline & $\geq 501$ & $40(8)$ \\
\hline \multirow[t]{3}{*}{ Time on ART (months) } & $6-48$ & $95(19.1)$ \\
\hline & $49-72$ & $105(21.1)$ \\
\hline & $73-156$ & $298(59.8)$ \\
\hline \multirow[t]{2}{*}{ ART drug Adherence } & Good & $373(74.9)$ \\
\hline & Fair and Poor & $125(25.1)$ \\
\hline \multirow[t]{2}{*}{ BMI } & Undernourished & $116(23.3)$ \\
\hline & Well-nourished & $382(76.7)$ \\
\hline
\end{tabular}

The magnitude of first-line ART virologic failure

243 The magnitude of virologic failure ( $\geq 1000$ RNA copies per ml) was found in $51(10.24 \%$; $95 \%$

244 CI: $7.57 \%, 12.91 \%)$. Since the start of HAART, $43(8.63 \%)$ of them encountered virologic

245 failure within 73-156 months (Table3). 
247 Table 3: Treatment failure after initiation of HAART in HIV/AIDS clients in Debre-Berhan 248 referral hospital and Mehal-Meda hospital, North Shoa Zone, April/2019 (n=498).

\begin{tabular}{|l|l|l|}
\hline Variable & Categories & Frequency (\%) \\
\hline Immunologic failure & Yes & $47(9.4)$ \\
\cline { 2 - 3 } & No & $451(90.6)$ \\
\hline Virologic failure & Yes & $51(10.2)$ \\
\cline { 2 - 3 } & No & $447(89.8)$ \\
\hline Months from ART initiation & $6-48$ & $3(5.88)$ \\
\cline { 2 - 3 } & $49-72$ & $5(9.8)$ \\
\cline { 2 - 3 } & $73-156$ & $43(84.4)$ \\
\hline
\end{tabular}

Factors associated with virologic treatment failure

250 After conducting bivariable analysis on predictor variables with a $\mathrm{p}$-value $\leq 0.2$, then 251 multivariable logistic regression analysis has been conducted to control the effects of socio252 demographic, behavioral, and clinical factors. Then, poor ART drug adherence $(\mathrm{AOR}=4.54$; $25395 \%$ CI: $2.09,9.87)$, people who had CD4 counts less than 250 cell $/ \mu 1(\mathrm{AOR}=24.88 ; 95 \% \mathrm{CI}$ : $25411.73,52.81)$ and PLWHA who had poor quality of life (QoL) (AOR = 2.65; 95\% CI: 1.12, 255 6.25) were found statistically associated with first-line ART virologic treatment failure (Table 256 4). 
263 Table 4: Bivariable and Multivariable Logistic Regression analysis of selected factors of

264 virologic failure in Debre Berhan and Mehal-Meda hospitals, North Shoa Zone, 2019 (n=498).

\begin{tabular}{|c|c|c|c|c|c|}
\hline \multirow{2}{*}{$\begin{array}{l}\text { Variables } \\
\text { Age(years) }\end{array}$} & \multicolumn{2}{|c|}{$\begin{array}{c}\text { Virologic } \\
\text { treatment failure }\end{array}$} & \multirow{2}{*}{ COR, $95 \% \mathrm{CI}$} & \multirow[t]{2}{*}{$\begin{array}{c}\text { p- } \\
\text { value }\end{array}$} & \multirow{2}{*}{ AOR, 95\% CI } \\
\hline & No & Yes & & & \\
\hline$<30$ years & 57 & 11 & $1.88(0.913,3.877)$ & 0.087 & - \\
\hline$\geq 30$ years & 390 & 40 & 1 & & \\
\hline \multicolumn{6}{|l|}{ Monthly Income } \\
\hline$<1500 \mathrm{ETB}$ & 262 & 35 & $1.545(0.830,2.873)$ & 0.170 & - \\
\hline$\geq 1500 \mathrm{ETB}$ & 185 & 16 & 1 & & \\
\hline \multicolumn{6}{|l|}{ Living area } \\
\hline Urban & 379 & 39 & 1 & & \\
\hline Rural & 68 & 12 & $1.715(0.855,3.442)$ & 0.129 & - \\
\hline \multicolumn{6}{|l|}{ ART adherence } \\
\hline Good & 385 & 25 & 1 & & 1 \\
\hline Poor & 62 & 26 & $6.458(3.505,11.89)$ & $<0.001$ & $4.54(2.09,9.87)$ \\
\hline \multicolumn{6}{|l|}{ Current BMI } \\
\hline$<18.5$ & 96 & 15 & 1 & & \\
\hline$\geq 18.5$ & 351 & 36 & $1.523(0.801,2.899)$ & 0.152 & - \\
\hline \multicolumn{6}{|c|}{$\begin{array}{l}\text { WHO stage of } \\
\text { HIV/AIDS }\end{array}$} \\
\hline Stage I & 442 & 42 & $18.94(6.07,59.11)$ & $<0.001$ & - \\
\hline Stage II \& III & 5 & 9 & 1 & & \\
\hline \multicolumn{6}{|c|}{ Current CD4 count } \\
\hline$<250$ cell $/ \mu \mathrm{l}$ & 25 & 34 & $33.76(16.62,68.88)$ & $<0.001$ & $24.88(11.73,52.81)$ \\
\hline$\geq 250 \mathrm{cell} / \mu \mathrm{l}$ & 422 & 17 & 1 & & 1 \\
\hline \multicolumn{6}{|l|}{ Quality of life } \\
\hline Poor & 237 & 42 & $4.15(1.97,8.73)$ & $<0.001$ & $2.65(1.12,6.25)$ \\
\hline Good & 210 & 09 & 1 & & 1 \\
\hline
\end{tabular}

\section{Discussion}

266 The identification and management of virological ART treatment failure is a key challenge for

267 HIV programs in resource-limited settings. ART treatment failure is a serious emerging threat to

268 the global scale-up of HIV treatment access and staying with this failing first-line therapy is 269 associated with an increased risk of morbidity and mortality (22). This study

270 particularly designed to determine the magnitude of virologic treatment failure $\quad(10.24 \%)$

271 and identify the predictors of virologic failure (Poor ART drug adherence, CD4 count less

272 than 250 cell/ $\mu 1$ and clients' whose poor quality of life). 
273 The size of virological treatment failure was 10.24\% (51/498). This finding was consistent with 274 the study conducted in guinea 13.7\% (23) and Bahir Dar 10.7\% (24). However, when compared 275 with other studies, for instance, a study conducted in coastal Kenya (24, 6\%) 276 (25), Debre Markos hospital (21\%) (26), Colombia (29\%) (15), Sweden, Honduran (74\%) (16), 277 and China (28\%) (17), it was low. This discrepancy may be due to most clients on first-line ART 278 treatment failure shift to second-line ART. This $10.2 \%$ virological treatment failure indicated 279 that first-line ART treatment may not be effective, as WHO recommends changing their first-line 280 ART regimen if levels of Non-nucleoside reverse-transcriptase inhibitor (NNRTI) pretreatment 281 HIV drug resistance (PDR) reach 10\%(27), so further investigation is needed on 282 pretreatment HIVDR to NNRTIs.

283 The current study showed that ART treatment adherence was the independent reason for 284 the virologic treatment failure and patients with poor ART drug adherence were 4.5 times at 285 higher risk of developing treatment failure compared to patients with good ART drug adherence. 286 This finding is consistent with a systematic review conducted in Ethiopia (28). Medication 287 adherence has an opportunity for best viral suppression, immune recovery, and as a result clinical 288 benefit will be gained. Thus, successful ART treatment requires all medications should be taken 289 as per prescribed by the physicians (health care service giver). However, this result contradicts 290 from studies done in the Tigray region of northern Ethiopia, Gondar and Felege-Hiwot Referral 291 Hospitals $(14,29,24)$. These variations may be related to the type of data collection method. In 292 our study, the data on adherence were collected from one-month recall self-reported missed 293 doses, and not directly collecting from the patients' charts.

294 Another predictor variable which has a significant association with virological failure 295 was CD4 count. Individuals who have CD4 count less than 250 cell/ $\mu 1$ were 24 times more likely 296 to develop virological failure than their counterparts (AOR $=24.88 ; 95 \%$ CI: 11.449, 52.81). 297 This result was in line with studies conducted in different parts of the world; University of 298 Gondar Hospital (AOR = 9.03; 95\% CI: 4.40, 18.50) (30), Zewditu Memorial Hospital (AOR = 299 2.67; 95\% CI: 1.29, 5.51) (31), a multi-center study conducted at three selected Hospitals 300 Northwest Ethiopia (AHR = 2.0; 95\% CI: 1.20, 3.50) (32), and Felege-Hiwot Hospital (AOR = 3018.63 ; 95\% CI: 3.32, 22.42) (24). Since ART treatment provides opportunities for viral load 302 suppression, recovery of immunity, and a client gets clinical benefits. Thus, all individuals taking 
ART drugs are expected to take all medications as prescribed. As a consequent, the number of CD4 counts increases and helps in the success of immunological treatment.

Health-Related Quality of Life (HRQoL) was another independent predictor variable for virologic treatment failure. Clients with poor Quality of Life were 2.6 times more likely to develop virologic failure than clients with $\operatorname{good}(\mathrm{QoL})(\mathrm{AOR}=2.65 ; 95 \% \mathrm{CI}: 1.12,6.25)$. Maintaining and improving the quality of life among people in ART follow up clinics is regarded as the most important component of HIV/AIDS care and treatment even though this concept is not given emphasis in developing countries where resources are scarce. Similar finding was observed in a study done in Ethiopia where immunologic treatment failure was associated with health-related quality of life among people infected with HIV/AIDS (33). Since measuring the quality of life of people with HIV/AIDS consisted of several dimensions (physical, psychological, independence, social, environmental, and spiritual), stakeholders should work on the betterment of the quality of life among people on HAART. As the result indicated $56.02 \%(51.65 \%, 60.40 \%)$ of the clients' quality of life was compromised by the disease (HIV/AIDS) and related complications. Thus, this significant number of clients needs their health-related quality of life to be changed.

\section{Strength and limitations of the study}

Since the study was conducted in health facilities taking a representative sample, the results can be generalized to health facilities in North Shoa Zone of Amhara Region, Ethiopia. But, the results are interpreted with limitations. First, due to the cross-sectional nature of the study design, we could not establish the temporal relationship between virological failure and its predictors. Recall and social desirability bias where the participants may not respond correctly for some sensitive questions due to memory loss and social norms. To reduce such systematic errors, during data collection period data collectors tried to explain the importance of honest response. Moreover, smaller frequency in certain categories of predictor variables may reduce the precision of the measure of association. Thus, the use of this study finding for any concern should be accounted in consideration having these inherent limitations of the study.

\section{Conclusion}


331 The magnitude of virological failure in this study was relatively high. Poor ART drug 332 adherence, $\mathrm{CD} 4$ counts less than $250 \mu \mathrm{l} / \mathrm{ml}$ and poor health-related quality of life were found to 333 be significant predictors of virologic treatment failure. Thus, Early ART failure detection is one 334 of the most important key improvement areas of the health care providers with close follow up of

335 the patients. Moreover, interventions that enhance the quality of life of clients on ART should 336 be established. Behavior change communication on the benefits of good ART drug 337 adherence should be strengthened which in turn expected to reduces the risks of the decrement 338 of CD4 counts.

339 List of Acronyms and abbreviation

340 ADR: Acquired HIV drug resistance; ART: Antiretroviral Therapy; ARV: Anti-retroviral

341 HAART: Highly Active Antiretroviral Therapy; HIV/AIDS: Human Immune Deficiency 342 Virus/Acquired Immune Deficiency Syndrome; HIVDR: HIV drug resistance; PDR: 343 Pretreatment HIV drug resistance; PLHIV: People Living with HIV; TDR: Transmitted HIV 344 drug resistance

\section{Declarations}

346 Acknowledgement

347 We would like to express our heartfelt gratitude to Debre Berhan University, College of Health 348 Sciences, and Department of Public Health for monitoring and evaluation of this project. This 349 work is impossible without the support of staff working at Debre Berhan Comprehensive 350 Specialized Hospital and Mehal Meda Hospital - thanks for your support during data collection 351 time. Moreover, study participants and data collectors deserve appreciations.

352 Funding

353 Not applicable 


\section{Competing interest}

355 The authors declare that they have no competing interest.

356 Availability of data

357 The data used to support the findings of this study are included in this manuscript.

358 Consent for publication

359 All authors agree to publish this article at AIDS RESEARCH AND THERAPY journal; part of 360 Springer Nature.

361 Authors' contribution

362 All stated authors have participated in this research project: BTD and BS conceptualized, 363 designed the study including data analysis, and manuscript writing; AD and EAB drafted the 364 manuscript and critically reviewed the manuscript. All authors read and approved the final 365 version of the manuscript.

\section{Ethics approval and consent to participate}

367 Ethical clearance was obtained from Debre Berhan University, College of Health Sciences, and 368 Ethical Review Committee. Official letter was submitted to Debre Berhan Comprehensive 369 Specialized Hospital and Mehal- Meda Hospital to inform and support data collection process. 370 Verbal informed consent was gained from the study participants by explaining the potential 371 benefits of the study. Moreover, participants were told that they can stop interview at any point 372 during data collection if they are uncomfortable and if they don't want to give answer for a 373 particular question. 


\section{References}

375 1. UNAIDS W. Fact Sheet: World AIDS Day 2017. Global HIV statistics. 2017.

376 2. Global HIV and AIDS statistics 2017-09-01.

377 3. EHNRI. HIV Related Estimates and Projections for Ethiopia. Addis Ababa: FMoH. 2012:6$378 \quad 14$.

379 4. UNAIDS D. Global Statistics. 2017.

380 5. Organization WH. Global Action Plan on HIV drug resistance 2017-2021. 2017.

381 6. HIV/AIDS JUNPo. Ending AIDS: Progress towards the 90-90-90 targets. Global AIDS $382 \quad$ Update. 2017.

383 7. Fund G, Organization WH. HIV drug resistance report 2017. 2017.

384 8. World Health Organization. Consolidated guidelines on the use of antiretroviral drugs for 385 treating and preventing HIV infection: recommendations for a public health approach: World 386 Health Organization; 2016.

9. World Health Organization. Global report on early warning indicators of HIV drug resistance: technical report. 2016.

10. Fethia K, Seble M. National Guidelines For Comprehensive HIV Prevention, Care And Treatment, 2017.

11. World Health Organization. Tackling HIV Drug Resistance:Trends, Guidelines and Global Action. 2017.

12. Gupta RK, Gregson J, Parkin N, Haile-Selassie H, Tanuri A, Forero LA, et al. HIV-1 drug resistance before initiation or re-initiation of first-line antiretroviral therapy in low-income and middle-income countries: a systematic review and meta-regression analysis. The Lancet infectious diseases. 2018;18(3):346-55.

13. Mpondo BC, Kilonzo SB, Meda JR, Gunda DW. Prevalence and predictors of Immunological failure among HIV-infected adults on HAART in Northwestern Tanzania: A cross sectional study. Tanzania Medical Journal. 2015;27(1).

14. Hailu GG, Hagos DG, Hagos AK, Wasihun AG, Dejene TA. Virological and immunological failure of HAART and associated risk factors among adults and adolescents in the Tigray region of Northern Ethiopia. PloS one. 2018;13(5):e0196259. 
15. De La Hoz JM, Bolaño L, Cárdenas O, González R, Sabbag J, Palacio L, et al. Characterization of treatment failure in HIV positive patients in the Colombian Caribbean region. Colombia Médica. 2014;45(4):162-7.

16. Murillo W, De Rivera I, Parham L, Jovel E, Palou E, Karlsson A, et al. Prevalence of drug resistance and importance of viral load measurements in Honduran HIV-infected patients failing antiretroviral treatment. HIV medicine. 2010;11(2):95-103.

17. Ma Y, Zhao D, Yu L, Bulterys M, Robinson ML, Zhao Y, et al. Predictors of virologic failure in HIV-1-infected adults receiving first-line antiretroviral therapy in 8 provinces in China. Clinical infectious diseases. 2010;50(2):264-71.

18. Debre Berhan referral Hospital, Monthly Facility Anti-retroviral Theraphy Report. December 2018.

19. Mehal Meda Hospital, Monthly facility ART Report. December 2018.

20. World Health Organization. Guidance on operations and service delivery: adherence to ART. 2013.

21. World Health Organization. Mental Health: Evidence and Research Department of Mental Health and Substance Dependence. World Health Organization, Geneva, 2002.

22. Avert. Global information and education on HIV and AIDS. 2017.

23. Gare J, Kelly-Hanku A, Ryan CE, David M, Kaima P, Imara U, et al. Factors influencing antiretroviral adherence and virological outcomes in people living with HIV in the highlands of Papua New Guinea. PLoS One. 2015;10(8):e0134918.

24. Bokretsion GB, Endalkachew N, Getachew KA. HIV/AIDS treatment failure and its determinant factors among first line HAART patients at Felege-Hiwot Referral Hospital, Bahir Dar, Northwest Ethiopia. Journal of AIDS and Clinical Research. 2017;8(11).

25. Hassan AS, Nabwera HM, Mwaringa SM, Obonyo CA, Sanders EJ, de Wit TFR, et al. HIV1 virologic failure and acquired drug resistance among first-line antiretroviral experienced adults at a rural HIV clinic in coastal Kenya: a cross-sectional study. AIDS research and therapy. 2014;11(1):9.

26. Yayehyirad AM, Mamo WT, Gizachew AT, Tadesse AA. Rate of immunological failure and its predictors among patients on highly active antiretroviral therapy at Debremarkos hospital, Northwest Ethiopia: a retrospective follow up study. Journal of AIDS and Clinical Research. $2013 ; 4(5)$. 
27. World Health Organization. Guidelines on the public health response to pretreatment HIV drug resistance: July 2017. 2017.

28. Aklilu Endalamaw, Mengistu Mekonen, Demeke Geremew, Fekadu Ambaw, Hiwot Tesera, Tesfa Dejenie Habtewold. Evidence that poor HAART adherence has a great impact on HIV/AIDS treatment failure more than severity of illness and opportunity of infection in Ethiopia: Systematic review and meta-analysis. bioRxiv preprint first posted online Oct. 11, 2018; doi: http://dx.doi.org/10.1101/440743

29. Ayalew MB, Kumilachew D, Belay A, Getu S, Teju D, Endale D, et al. First-line antiretroviral treatment failure and associated factors in HIV patients at the University of Gondar Teaching Hospital, Gondar, Northwest Ethiopia. HIV/AIDS (Auckland, NZ). 2016;8:141.

30. Belete Bayu, Amare Tariku, Abera Balcha Bulti, Yohannes Ayanaw Habitu, Terefe Derso, Destaw Fetene Teshome. Determinants of virological failure among patients on highly active antiretroviral therapy in University of Gondar Referral Hospital, Northwest Ethiopia: a casecontrol study. HIV/AIDS - Research and Palliative Care. 2017:9 153-159

31. Sisay C, Bekele A, Sisay A, Mekonen H, Terfa K. Incidence and Predictors of AntiRetroviral Treatment (ART) Failure among Adults Receiving HIV Care at Zewditu Memorial Hospital, Addis Ababa, Ethiopia. J AIDS Clin Res. 2017;8(749):2.

32. Tsegaye AT, Wubshet M, Awoke T, et al. Predictors of treatment failure on second-line antiretroviral therapy among adults in northwest Ethiopia: a multicenter retrospective followup study. BMJ Open 2016; 6:e012537. doi:10.1136/bmjopen-2016-012537

33. Kebede Abera, Teferi Gedif, Ephrem Engidawork, Tsige Gebre-Mariam. Quality of life of people living with HIV/AIDS and on highly active antiretroviral therapy in Ethiopia, African Journal of AIDS Research, 2010; 9:1, 31-40. doi.org/10.2989/16085906.2010.484560 